\title{
Gold Nanoparticles A Renaissance in Gold Chemistry
}

\author{
Robin Whyman \\ Department of Chemistry, \\ University of Liverpool, \\ PO Box 147, Liverpool, L69 3BX, UK
}

\begin{abstract}
Vapour synthesis techniques have been used to prepare nanoparticulate dispersions of gold and other precious metals in non-aqueous solvents. The dimensions of these solvent-stabilised particles, which can be controlled within the 1-3nm size regime, effectively encompass the areas of molecular chemistry (as typified by high-nuclearity metal clusters) and the smaller colloidal metals. Gold nanoparticles differ from those of the other metals in exhibiting unusual time-and concentration-dependent behaviour. A regime of preparative conditions under which 1-3nm size gold particles, which are stable with respect to aggregation as a function of time, is defined. Some implications for these new developments are indicated.
\end{abstract}

The chemistry of gold and its complexes has been a subject of continuing interest for many years (1), with steady but in general less than dramatic progress. A notable exception to this concerns the preparation and characterization of a wide range of cluster compounds of gold having various nuclearities and structures (2). Very recently, however, other aspects of gold chemistry are beginning to undergo a resurgence of interest, largely as a result of chance discoveries by interdisciplinary research groups, seeking potential applications in areas such as nanotechnology, self-assembly, catalysis and molecular electronics.

Colloidal gold has attracted much attention ever since Faraday's early work on the reduction of an aqueous gold salt with phosphorus in carbon disulfide which gave a ruby coloured aqueous solution of dispersed gold particles $(3,4)$. In common with other groups (5), we have been interested in the preparation, characterization and properties of small metal particles, in particular those which comprise that intermediate state of matter which encompasses molecular (metal cluster) and bulk (colloidal metal) chemistry. Such materials frequently display unusual physical (structural, electronic, magnetic, and optical) and chemical (catalytic) properties and are therefore of considerable fundamental interest.

The natural progression from a cluster to the smallest colloidal metal particle may occur in a continuous fashion although the situation is complicated by the fact that high nuclearity clusters are usually prepared and studied in organic solvents whereas colloidal metal preparations usually involve the reduction of aqueous solutions of metal salts. One of the main hindrances to progress in this region which encompasses molecular chemistry and the colloidal state has been the paucity of relatively simple, reproducible and generally applicable synthetic procedures, together with difficulties associated with structural characterization. In order to address the synthesis problem we have utilized recent developments in metal vapour synthesis (MVS) (6) and have demonstrated that the electron beam evaporation of Groups 8 to 10 (VIII) and Group 11 (IB) metals followed by co-condensation with the vapours of organic solvents at $77 \mathrm{~K}$ leads, after warm-up, to the formation of solvent-stabilized metal nanoparticles, the dimensions of which can be controlled within the critical 1-5 nm particle size range. In the presence of suitable dispersing agents particles in even narrower size ranges $(1-3 \mathrm{~nm})$ can be generated and stabilized (7). The combinations of metals and organic molecules which have been investigated include ruthenium, rhodium, palladium, platinum and silver, as well as gold, and methylethylketone (MEK), acetone, toluene and methylcyclohexane. Typical concentrations of the solvent-stabilized nanoparticles are quite high (relative to aqueous systems) and, for third row metals, lie in the range $3.5 \times 10^{-3}$ to $1.5 \mathrm{x}$ $10^{-2}$ molar; based on the mass of metal evaporated.

A general feature of the preparations of solvent-stabilized platinum group metal nanoparticles is the formation of brown solutions with colour intensities proportional to the amount of metal evaporated and the 


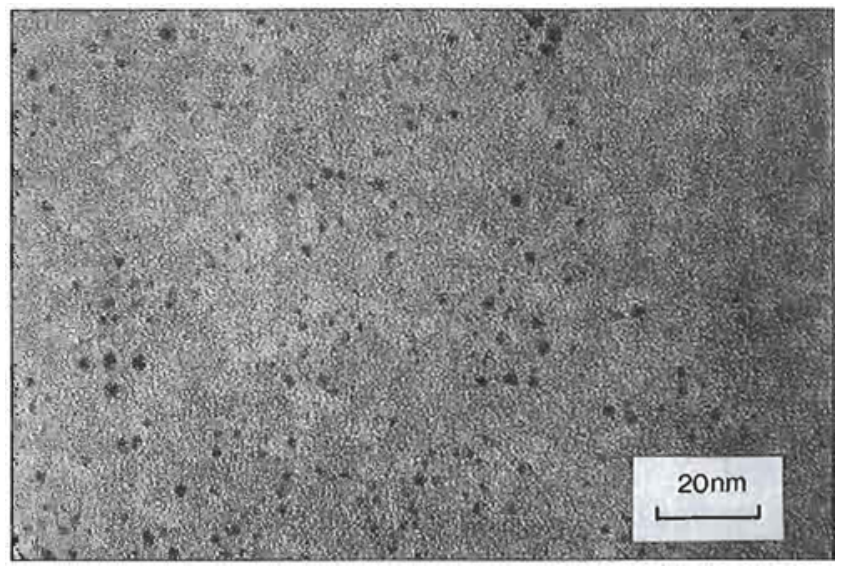

Figure 1 Typical electron micrograph of gold nanoparticles (ex-Au/MEKIKDI) dispersed on lacey carbon film

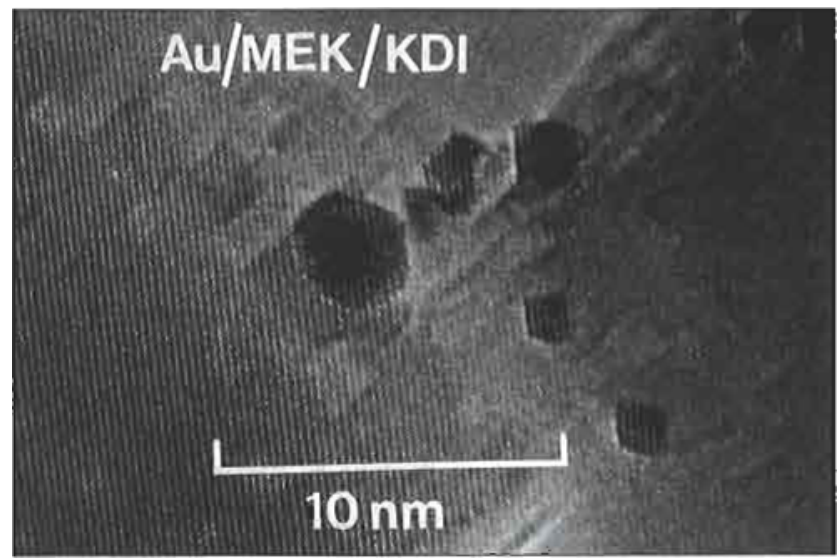

Figure 2 Octahedral and cuboctahedral gold particles (exAu/MEK/KDI) exhibiting an epitaxial relationship with the magnesium oxide smoke cube support; imaged along the [110] direction

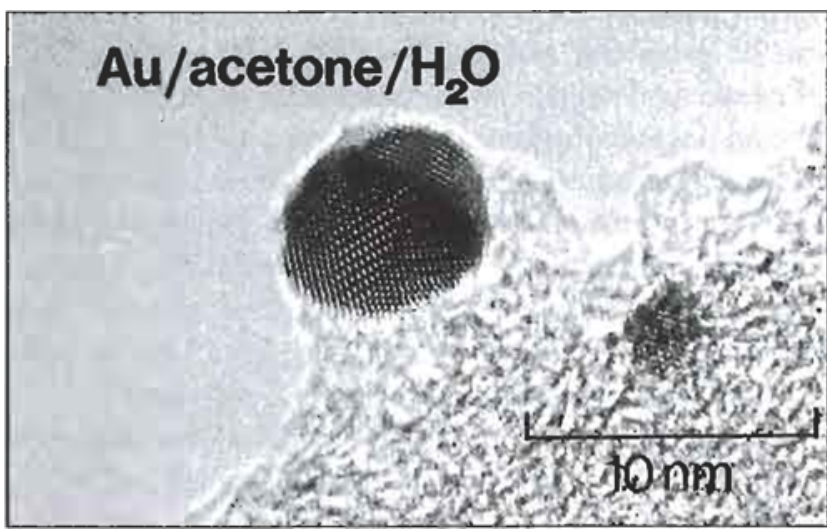

Figure 3 Multiply twinned gold particles ex-Aulacetone/water shade of brown varying slightly with the nature of the metal. For example, ruthenium-containing solutions appear yellow-brown, rhodium reddish-brown and palladium and platinum both dark brown. In general, MEK solutions are stable with respect to storage under dinitrogen over relatively short periods (days - weeks), but they change slowly over 3-6 months with the gradual deposition of solid and a slight reduction in intensity of the colour of the supernatant liquid. The use of dispersing agents enhances the stability of the solutions and reduces the rate of deposition of solids. In terms of the different solvents used the stability of the nanoparticles with respect to long term storage lies in the order MEK > acetone/water $>$ toluene $>$ methylcyclohexane.

Comparison of nanoparticles of the coinage group metals with the platinum group metals highlights some significant differences. Silver nanoparticles obtained by the co-condensation of Ag with MEK seemed particularly unstable with respect to flocculation/precipitation, irrespective of the presence of dispersing agents. Solutions of gold nanoparticles displayed an unusual concentration-dependent aggregarion phenomenon which could be monitored using a combination of optical spectroscopy and high-resolution transmission electron microscopy (HRTEM), but which did not result in precipitation.

In general, factors which qualitatively control the particle sizes include the mass of metal evaporated, the rate of evaporation, the metal/co-condensate ratio and the co-condensation temperature. Low values of all of these favour the formation of smaller particles. Factors such as the rate of matrix warm up and warm up under a static vacuum or an atmosphere of dinitrogen can also be significant. The nanostructures have been characterized by HRTEM and evidence for fcc octahedral, fcc cuboctahedral and icosahedral metal particles obtained; quasi-molten states have also been observed in a number of these systems. In addition to discrete particles, larger aggregates of the small primary particles have been observed, some of which appear to be organometallic in nature.

Solvent-stabilized Au nanoparticles prepared by the MVS route show some significant differences from the platinum group metals. Small particles in the $1-3 \mathrm{~nm}$ size range may also be prepared and characterized, as illustrated in Figure 1. At higher magnifications the nanoparticles derived from Au/MEK/KDI (KDI is a polymeric dispersant), when dispersed on $\mathrm{MgO}$ smoke cubes, clearly exhibit an epitaxial relationship with the $\mathrm{MgO}$ support material (Figure 2). However, in addition to small particles, these MVS preparations can also result in the formation of much larger particles (up to $20 \mathrm{~nm}$ in size) even 
when the preparations have included dispersing agents. Figures 3 and 4 illustrate examples of multiply twinned particles obtained from Au/acetone/water preparations. Moreover, in some, but not all, cases the particle sizes have been found to vary with storage, as have the colours of their solutions. Those containing the smallest particles $(1-3 \mathrm{~nm})$ are brown whereas those containing particles larger than $5 \mathrm{~nm}$ exhibit purple/red colourations and show the plasmon absorption characteristic (8) of metalllic gold at ca $540 \mathrm{~nm}$ in the optical spectrum.

\section{CONCENTRATION-DEPENDENT GROWTH PHENOMENON ASSOCIATED WITH GOLD NANOPARTICLES}

The particle growth phenomenon was first observed as a consequence of changes in the colour of some of the solutions from brown to red and/or purple during storage over a period of time. Figure 5 shows typical examples of the changes occurring in the optical spectra, in particular the growth of the plasmon absorption at ca $540 \mathrm{~nm}$ as a function of time. Initially this particle growth phenomenon appeared to occur almost randomly, but careful examination of what is now quite an extensive body of information has allowed the following rationalizations in respect of these Au/MEK preparations:

(i) Immediately after preparation and warm up in the MVS reactor, brown solutions containing primary particles in the size range 1-4 $\mathrm{nm}$ are obtained.

(ii) The primary particle size appears to be dependent upon the metal concentration; in general, high concentrations favout larger particle sizes. Rapid warm-up has been found to enhance the relative amounts of smaller particles, possibly by a quenching process.

(iii) The use of dispersing agents has little effect on the primary particle sizes but their presence prevents the aggregation/chaining of individual particles, a phenomenon which appears to be associated with the development of blue colours in the solutions.

(iv) Below a critical concentration (ca $0.01 \% \mathrm{wt} / \mathrm{v}$ ) the primary particles are unstable with respect to storage at room temperature. Over a period of weeks, larger particles appear to grow at the expense of smaller particles with the concomitant development of red or purple colours. Storage of the solutions at $-20^{\circ} \mathrm{C}$ significantly slows but does

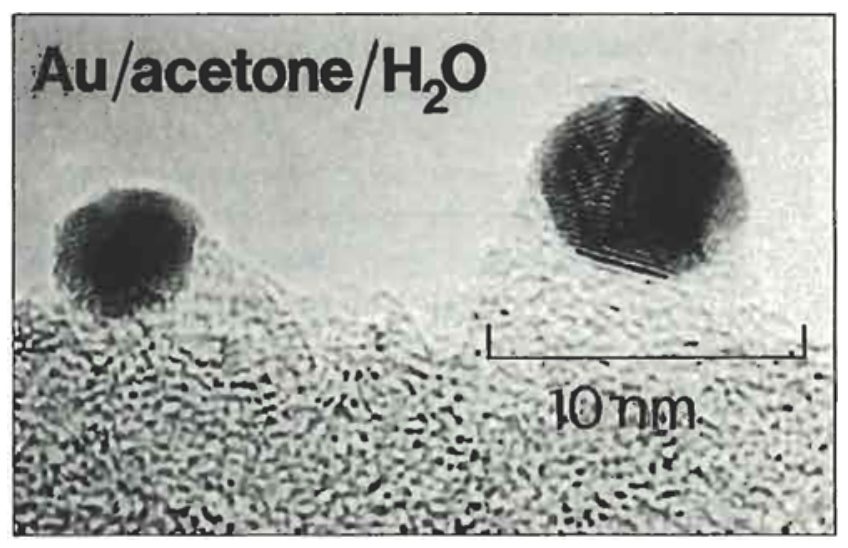

Figure 4 Multiply twinned gold particles ex-Aulacetone/water

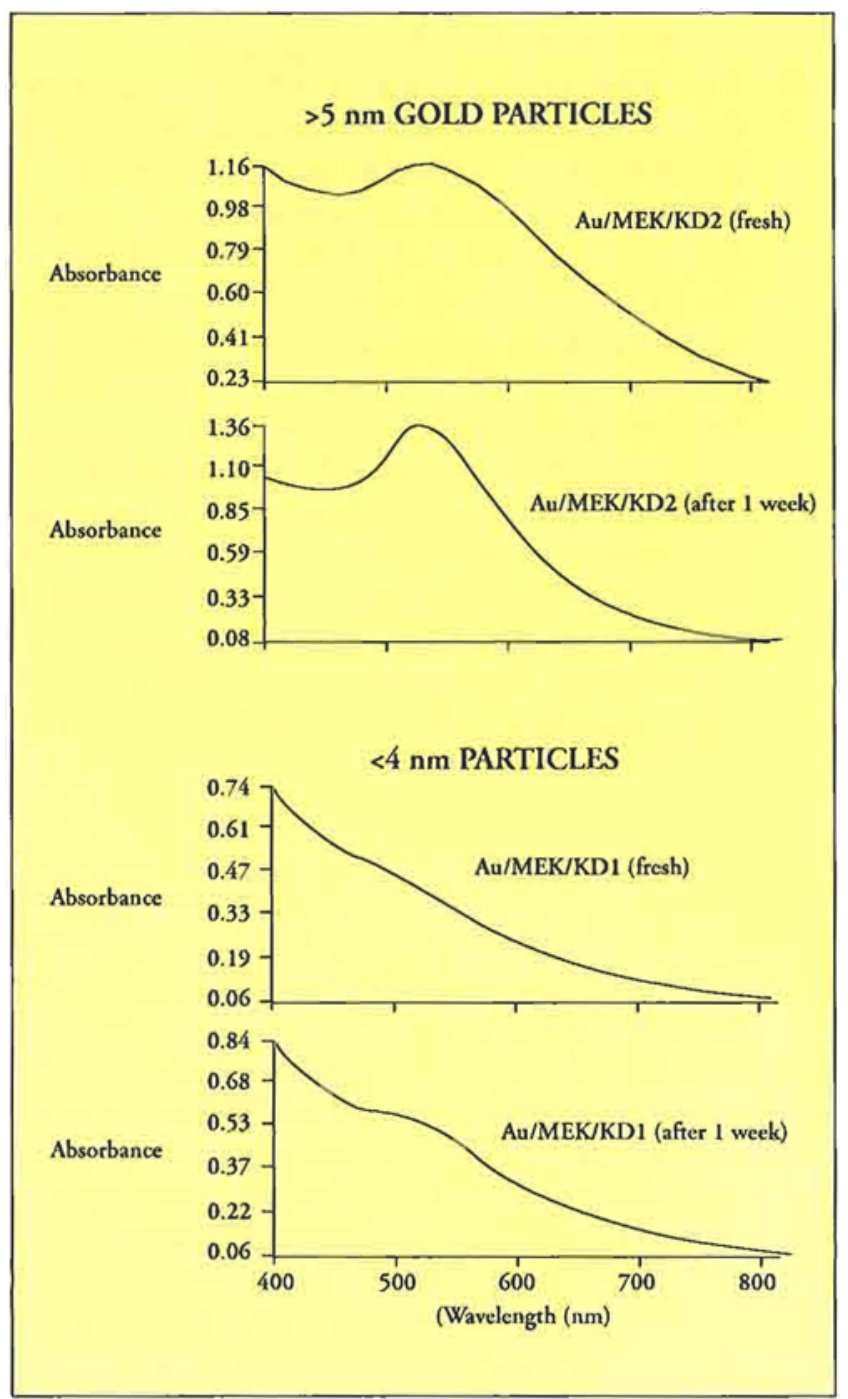

Figure 5 Typical optical absorption spectra of gold nanoparticles 
not completely inhibit the rate of particle growth.

(v) Above the limiting concentration, i.e. $>0.01 \%$ $\mathrm{wt} / \mathrm{v}$, the primary particles appear stable with respect to storage at room temperature over a period of months, the solutions remaining brown in colour. However, upon dilution (to below the limiting concentration) by the addition of pure solvent, particle growth is initiated and then occurs at an equivalent rate to that observed with corresponding samples containing the same initial concentrations below $0.01 \% \mathrm{wt} / \mathrm{v}$ (see Figure 6). A parallel change in colour from brown to red (or purple) is also observed. The explanations for these unusual effects are not obvious. For example, one possible explanation concerning desorption of the dispersing agent on dilution can be eliminated because the effect is also observed in the absence of added dispersing agents.

(vi) The plasmon absorption at $520-540 \mathrm{~nm}$ in the optical spectrum, which is associated with typical preparations of colloidal gold, and which is responsible for their red/purple colours, is not observed in spectra of the freshly prepared materials. As particle growth occurs, this band becomes evident and its appearance seems to correspond with the presence of particles $>4 \mathrm{~nm}$ in size. However, there appears to be no obvious relationship between the measured absorbance of this band and particle size in the range $4-30 \mathrm{~nm}$. This may arise as a consequence of the fact that the larger particles are no longer perfectly spherical in shape but are partially elongated or aggregated and this effect is superimposed on the plasmon band width size effect. Above $30 \mathrm{~nm}$, precipitation of the particles occurs.

(vii) Initial experiments with other solvents, e.g. acetone, acetone/water mixtures, methylcyclohexane and toluene, indicate that the behaviour described above may be quite general although the detailed parameters are different and require further investigation.

(viii) In order to reproducibly obtain $1-3 \mathrm{~nm}$ size $\mathrm{Au}$ nanoparticles which are stable with respect to aggregation, it is necessary to work above the limiting concentration level of $0.01 \% \mathrm{wt} / \mathrm{v}$ and to employ faster rather than slower warm-up techniques.

Thus, we have established a regime of preparative conditions under which 1-3 nm size Au particles, which are stable with respect to aggregation and growth, and which do not display the plasmon absorption characteristic of most colloidal gold preparations, may be produced.

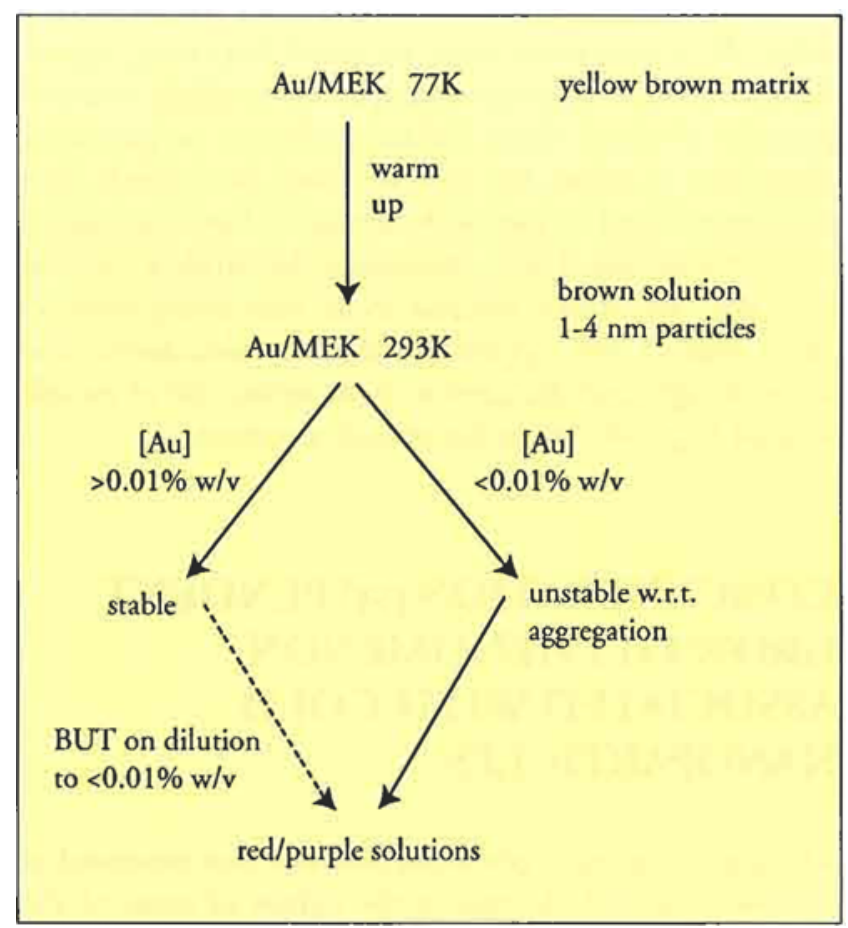

Figure 6 Gold Particle Growth Phenomena

\section{APPLICATIONS AND FUTURE DEVELOPMENTS}

Now that reproducible methods for the preparation of solvent-stabilized metal nanoparticles are available, it is appropriate to explore their properties and chemical reactivity in more detail. A promising area for investigation is that of catalysis, and we have been exploring the behaviour of Pt and Pd nanoparticles as catalysts for the enantioselective hydrogenation of ethyl pyruvate in the presence of cinchonidine as modifier (9). In preliminary investigations using gold nanoparticles we have demonstrated that they can provide active catalysts for the low temperature oxidation of carbon monoxide to carbon dioxide $(10,11)$.

Finally, it should be emphasized that MVS techniques do not represent the only preparative routes to sub-colloidal gold nanoparticles stabilized in organic solvents. We have recently reported a simple reductive route to thiol-derivatized gold nanoparticles using the borohydride reduction of chloroauric acid in the presence of alkane thiols (12). This is an exciting development because in some respects these materials behave as chemical compounds. Thus they are stable over extend- 
ed periods and can be precipitated, re-dissolved, chromatographed and size separated via fractional crystallization. Their functionalized exterior surfaces offer the prospect of controlled assembly into three dimensional arrays or networks which could find a wide range of applications in the fields of sensors and molecular electronics.

It can therefore be concluded that gold chemistry is undergoing a renaissance (13-16), and new developments of the kind highlighted here indicate potential for new applications which were certainly not foreseen ten years ago.

\section{ACKNOWLEDGEMENTS}

I wish to thank the Royal Society/SERC for an Industrial Fellowship and the SERC Nanotechnology Initiative for support.

\section{ABOUT THE AUTHOR}

Dr Whyman is Lecturer in Inorganic Chemistry at the University of Liverpool and Assistant Director of the Leverhulme Centre for Innovative Catalysis, having previously spent over 25 years with ICI working on a variety of 'leading edge' exploratory research topics related to the activation of molecules, especially in relation to their use in catalysis.

\section{REFERENCES}

1 R.J. Puddephatt, 'The Chemistry of Gold', Elsevier, Amsrerdam, 1978

2 D.M.P. Mingos, J. Chem. Soc. Dalton Trans., 1996,561 , and references therein
3 M. Faraday, Philos. Trans. Roy. Soc. London, 1857, 147,145

4 'Colloidal Gold: Principles, Methods and Applications', ed. M.A. Hayat, Academic Press, San Diego, 1989, Vol 1

5 See, for example, 'Clusters and Colloids', ed. G. Schmid, VCH, Weinheim, 1994

6 G.A. Ozin, M.P. Andrews, C.G. Francis, H.X. Huber and K. Molnar, Inorg. Chem., 1990, 29, 1068

7 R.W. Devenish, T. Goulding, B.T. Heaton and R. Whyman, J. Chem. Soc., Dalton Trans., 1996, 673

8 W.P. Halperin, Rev. Mod. Phys, 1986, 58, 533.

9 P.J. Collier, T. Goulding, J.A. Iggo and R. Whyman, 'Chiral-Reactions in Heterogenous Catalysis', eds. G. Jannes and V. Dubois, Plenum, New York, 1995, p. 105

10 G.J. Hutchings, M.R.H. Siddiqui and R. Whyman, to be published

11 M. Haruta, S.Tsubota, T. Kobayashi, H. Kageyama, M.J. Denet and B. Delmon, J. Catal., $1993,144,175$

12 M. Brust, M. Walker, D. Bethell, D.J. Schiffrin and R. Whyman, J. Chem. Soc., Chem. Commun., 1994,801

13 M. Brust, J. Fink, D. Bethell, D.J. Schiffrin and C. Kiely, J. Chem. Soc., Chem. Commun., 1995, 1655

14 D.V. Leff, P.G. Ohara, J.R. Heath and W.M. Gelbert, J. Phys. Chem., 1995, 99, 7036

15 R.L. Whetten, J.T. Khoury, M.M. Alvarez, S. Murthy, I. Vezmar, Z.L. Wang, C. Cleveland, W.D. Luedtke and U. Landman, Nature, submitted

16 R.H. Terrill, T.A. Postlethwaite, C.-H. Chen, C.-D. Poon, A. Terzis, A. Chen, J.E. Hutchison, M.R. Clark, G. Wignall, J.D. Londono, R. Superfine, M. Falvo, C.S. Johnson, E.T. Samulski and R.W. Murray, J. Am. Chem. Soc., 1995, 117, 12537 